\title{
Carlos Altamirano, historiador
}

Fernando J. Devoto

Universidad de Buenos Aires / Universidad Nacional de San Martín

Agradezco a Adrián Gorelik y al Centro de Historia Intelectual de la Universidad de Quilmes por haberme invitado hoy aquí a esta mesa redonda para presentar un libro de Carlos Altamirano. Este bello libro es una oportunidad, pero quizá no para reflexionar sobre él, sino sobre su autor, ya que es innegable que no estamos aquí por el libro o principalmente por él, cualesquiera sean sus méritos (y son muchos). Estamos aquí para brindar un homenaje a Carlos y para dar un testimonio de nuestra amistad y nuestra gratitud. Estamos aquí por la deuda que tenemos con Carlos y no solo en el terreno intelectual sino en el personal, porque además de un notable intelectual, lo que con ser mucho nunca es bastante, ha sido siempre una persona generosa. Creo que muchas de las muchas presencias que están hoy aquí testimonian lo primero, pero también lo segundo.

Un libro de recuerdos, o como se lo quiera llamar, implica siempre recortes. Algunos inherentes al género, otros inherentes a este caso. Desde luego que es inherente a las memorias de un autor que estén sometidas a sus recuerdos y sus olvidos, y a su voluntad de elegir lo que quiere decirnos y lo que no quiere decirnos, como ocurre con cualquier testimonio voluntario. Sin embargo, en cada testimonio hay más de lo que el autor ha querido explícitamente decir y buscarlo es, como se sabe, el oficio de los historiadores. Otro, más general, es que al organizar mentalmente en una secuencia aquellos recuerdos y al trasladarlos luego a otro formato con otras reglas como son los de una narración escrita con sus códigos específicos, se pierden también muchas cosas por el camino. Era un viejo argumento ya de Wilhelm von Humboldt.

Todavía tenemos, en relación con este caso, otros recortes. Libros, lecturas ¿Somos nuestras lecturas o lo que nos caracteriza son nuestras lecturas? Así formulada la pregunta yo diría que la respuesta es no. Pero si la hiciéramos de otro modo: ¿lo que escribimos es hijo de nuestras lecturas?, aquí diría sí, pero no solo. Las lecturas sí pero también, al menos, las relaciones sociales e intelectuales, por un lado, y las experiencias, por el otro. Un profesor que al salir de una clase o en un bar deja caer a un estudiante destacado un nombre como sugerencia de lectura, otra persona que escribe cartas de recomendación para el mismo, ahora ya joven profesor y al que los avatares de la política empujan a migrar a Buenos Aires, y que al hacerlo prefiguran itinerarios, ¿cómo entran en esta historia? Y me refiero acá a Carlos Giordano y a Hilda Torres y desde luego a Carlos Altamirano.

Somos también hijos de contingencias, azares, fortunas, y quisiera recordar una historia que no está en el libro. Siendo adolescente Carlos quería a toda costa poder conocer Buenos Aires. Para lograrlo, en esos años del primer peronismo, se anotó en todos los campeonatos deportivos posibles, atletismo, vóley, basquetbol, ajedrez, cuyo premio mayor era disputar las finales en Buenos Aires. ¿Y si hubiera descollado en alguno de ellos, las cosas hubieran sido diferentes? Claro está que nunca lo sabremos, pero podemos apelar a una reflexión del mismo Carlos en su excelente libro sobre Frondizi. Dice ahí sobre Frondizi. "Vale la pena consignar, sin embargo, la contingencia de que, en 1925, antes de cursar el último año del bachillerato, intentará ingresar en el Colegio Militar, sin lograrlo. El hecho indica que el proyecto vital que en definitiva fue el suyo no estaba trazado de antemano, como un destino".

Pero vayamos al libro y a la tarea que Adrián me encomendó. Notaría algo obvio, que el libro se llama "Estaciones" y esa palabra ya dice bastante. Nos introduce desde ella misma en la temporalidad y el cambio. Nos alude a muchos Altamiranos en Altamirano, pero ese devenir o ese pensarse históricamente no postula una ruptura radical entre los varios Altamirano o al menos la narración no la postula. Las estaciones son momentos en una secuencia que se propone unitaria: la unidad del yo, de la persona, de la sustancia, úsese la expresión que se quiera. Algo 
que es, por lo demás, una adquisición de la cultura occidental luego de un largo proceso. Por ello, colijo, estaciones y no, por ejemplo, transiciones. Pero soy consciente de que estoy tratando de conjeturar demasiado sobre un título.

De esas estaciones, a mí se me asignó la última. Aquella que corresponde con el cultor de la historia intelectual que coincide, aproximadamente, con la de su inserción en el mundo académico cuando, según nos cuenta, decidió seguir las sugerencias de Hilda Sabato y Marcelo Cavarozzi, primero en el CEDES y luego en el CONICET. Y noto al pasar que en este libro, a esta última estación, como él mismo la llama, aunque en su itinerario vital ocupa unos 35 años y concentra creo la mayor parte de su producción escrita, solamente le dedica 13 de las 126 páginas del libro. Una ventaja, si se quiere, para el comentarista, que tiene más libertad para fabular. En cualquier caso, esa decisión de dedicar más tiempo a la formación y al trayecto ascendente de una vida o de un proceso histórico tiene otros ejemplos en la obra de Altamirano. Nótese de nuevo el Frondizi, que culmina prácticamente en 1962 y al período posterior -treinta y tres añosle dedica, bajo el rubro "epílogo", dos páginas.

De todo lo que Carlos nos dice sobre sus estaciones precedentes, quisiera retener aquí tres cosas. La primera ya fue aludida: esa vocación o tentación por la ciudad como el lugar en el que se quiere desplegar el futuro y que constituye un itinerario no inusual, en etapas: desde un pequeño pueblo rural a la ciudad de provincia (Corrientes) y de esta a la gran ciudad (Buenos Aires). Y, sin embargo, por fuerte que fuese esa tentación, lo que decidió el pasaje o al menos el momento del pasaje, en 1967 durante el onganiato, fueron los avatares de la política argentina, que en sus coacciones, más oprimentes en un lugar de sociabilidad de cercanías como Corrientes, obligaban a emigrar.

La segunda es la voluntad, el esfuerzo, hacer las cosas bien hechas. Cito a Carlos de nuevo: "Tras la enseñanza que extraje de la revista (Cuadernos de Cultura) tuve que hacer después otros aprendizajes para convertirme en aquello que quería ser, un militante bien formado"... lo que implicaba muchas más lecturas además de asimilar el "canon". Y buena parte del libro muestra esa voluntad. Luego no sería solo un marxista bien formado, sino un historiador bien formado, y agregaría muy bien formado, lo que implicó otros muchos esfuerzos en ese contexto que es y ha sido el de los intelectuales latinoamericanos: autodidactismo y cultura de mezcla.

El tercer rasgo es la cultura comunista, a la que tantas páginas dedica Carlos en este libro y sobre el que con más pertinencia y competencia se ha hablado ya aquí. El inventario de los límites, debilidades y aun alucinaciones de esa tradición intelectual en la Argentina ya ha sido hecho demasiadas veces y en exceso. Podrían también señalarse algunos de los elementos positivos y sobre todo colocar el problema en contexto. Diría ante todo que era una cultura de o con libros, y en este país eso no era ni es poco. Empero, todavía más. Si colocada en Buenos Aires la cultura comunista difícilmente descollaba, colocada en otro contexto, una ciudad o un pueblo de provincia, su significado era muy otro. Bien podía ser que esa fuera la única existente. Pensé en esto cuando leí el libro de Carlos y, casi en paralelo, las crónicas de Paraná que Amaro Villanueva publicaba en el diario $E l$ Litoral de Santa Fe, desde comienzos de la década de 1940. Recordé también el pueblo donde vivía mi abuela en la provincia de Buenos Aires, donde el único que tenía libros y hablaba de ellos, según mi madre, era un comunista que pertenecía a una familia principal y que era quizás (o eso entendí) el único comunista del pueblo. En este punto -y en esos contextoscultura comunista quería decir cultura letrada tout court. Y en el contexto de esa cultura aparece a contraluz la figura de Agosti, quizás el que mejor encarnó para la generación de Carlos la figura del maestro que no quiso, no pudo, ser.

Empero veamos al historiador intelectual. Carlos nos ha facilitado el trabajo, ya que escribió un artículo: "Ideas para un programa de historia intelectual". Rescatemos la apertura porque es finalmente una apertura muy a la Altamirano, es decir no prescriptiva sino abierta: "la historia intelectual se practica de muchos modos", y agrega "lo que por otra parte, es semejante a lo que pasa en el conjunto de la práctica histórica. Dispersión teórica y pluralización de criterios", en sus palabras. En ese contexto el modo que propone Altamirano es ante todo explorar un territorio donde confluyen la historia política, la historia de las élites culturales y el análisis histórico de la literatura de ideas, y al hacerlo no olvida que se trata de ideas, pero no solo de ideas, y que los instrumentos para explorarlas se dilatan hacia la crítica 
literaria, por un lado, y hacia la sociología de las élites por el otro.

Una historia atenta a las conceptualizaciones, a los criterios a emplear, a los recortes y a las reflexiones formalizadas en diferentes grados de abstracción y generalización. Sin embargo, yo diría, hasta ahí. Como el mismo Altamirano nos recuerda en la presentación de un conjunto de ensayos de historia intelectual, citando a Roger Chartier: "proponerse cuestiones de definición en el terreno de la historia intelectual es entrar en dificultades". Por otra parte, ciertamente no ignoro que Carlos ha escrito mucho y bien, por ejemplo, sobre la figura del intelectual, definición, recortes y tipologías, sea en el contexto europeo, sea en el latinoamericano, así como ha reflexionado también sobre el empleo de palabras y categorías, y muy recientemente en Córdoba, en unas jornadas organizadas por Ana Clarisa Agüero, con una celebrada intervención sobre la noción "izquierda". Tampoco ignoro que en algunos trabajos, por ejemplo el admirable "La pequeña burguesía, una clase en el purgatorio", entremezcla referencias que podríamos llamar teóricas con un análisis de textos de intelectuales argentinos tratados como evidencia empírica, si se puede decir así. Sin embargo, mirada en conjunto, su producción histórica, siempre colocada en esta fase entre ideas y política, no asedia al lector con referencias teóricas o historiográficas (y debemos o mejor debo agradecérselo). Por el contrario, propone, en mi mirada, una narración cercana a los textos y si se quiere una operación que encierra algo muy viejo y ya olvidado: autor, texto y contexto. Y esto vale para los retratos de figuras individuales más allá de Frondizi, "el hombre de ideas como político", como los que dedicó a Sarmiento, Quesada, Romero, Halperin, Portantiero, y aquel que yo prefiero, pero esto es, claro está, arbitrario, que es el que en forma de una carta a Cesar Tcach evoca la figura de Héctor Schmucler, y que acaba de publicarse. Retratos "realistas" si se quiere, o que crean en el lector un fuerte efecto de realidad que deriva, imagino, de colocarlos en la tensión entre su vocación, de poder, de prestigio, redentoristas, lo que fuere, $y$ sus imposibilidades, personales o contextuales.

Y si en esa breve galería de personajes, y retomando la distinción de Ortega en su Mirabeau o el político, que el mismo Altamirano usa en algún lugar, hay un desbalance hacia los intelectuales, no es menos cierto que, en aquellas obras que analizan en conjunto un período o una época, el balance es más equilibrado y los políticos adquieren tanta relevancia como los hombres de ideas.

Qué decir de trabajos como los reunidos en Peronismo y cultura de izquierda (2001, pero que recopila trabajos de la década precedente), del largo ensayo preliminar a Bajo el signo de las masas (2001) o de "Pensar la Argentina entre dos centenarios" (2010). Lo primero que diría es que no han envejecido, lo que es bien significativo, en especial en el carrousel argentino y sobre todo, considerando la fecha de edición, los dos primeros volúmenes aludidos. ¿Por qué? Creo que precisamente porque no abundan ni en referencias teóricas ni en el uso de esos modelos que tan pronto se ponen de moda como quedan de lado. Y por lo demás ¿no es eso que se solía llamar aparato teórico lo que envejece más rápidamente? La segunda razón es que, en mi mirada, las lecturas de Altamirano se esfuerzan y logran ser, ¿cómo llamarlo?, "ecuánimes", entendiendo por eso la voluntad de dar a cada uno lo suyo y en esa voluntad no intentar satisfacer los instintos inmediatos, los apetitos voraces y mudables, au jour le jour, de los lectores.

No ignoro que Altamirano es un intelectual situado y tampoco lo ignora él, y aunque no lo recuerde demasiadas veces, lo hace las suficientes. Ahí está, por ejemplo, la referencia a Koselleck y la cuestión del perspectivismo, o la admisión de que, cito a Carlos: "el punto de partida de nuestra ponencia. Destinada a alegar, es decir, a citar y traer a favor de un propósito, como prueba o defensa, algunos hechos, argumentos y ejemplos, no tiene otra pretensión que la de esbozar un programa posible de trabajo". Alegar, retengamos la palabra. Empero, agreguemos un tercer ejemplo que muestra otro ángulo del problema, más específicamente argentino. En un trabajo sobre "el peronismo verdadero" dice Carlos: "nadie está en condiciones de hacer ironías sobre los corsi e ricorsi de la experiencia argentina". Ego primus peccavi, debería comenzar el credo de un intelectual argentino, o al menos, el mío.

Cierto, a veces le pedimos demasiado al historiador, como si le pidiésemos a un mago que muestre sus trucos permanentemente. Carlos lo hace de manera episódica, pero esa basta para un lector atento. Prefiere una narrativa neutral, distanciada, de vocación rankeana, en el significado descriptivo y positivo que puede 
atribuírsele a ese esfuerzo de lidiar consigo mismo, de esa imposible "objetividad" (pero que la objetividad no sea posible no quiere decir que la voluntad de objetividad no lo sea). Por lo demás, como se sabe, los historiadores estamos atrapados entre el hecho de que el pasado que conocemos lo creemos verdadero -y claro está, lo es para nosotros- y el saber que hay un punto de vista, o por decirlo con Weber, que en esto seguía a Rickert, una referencia a los valores, que está en el comienzo de toda investigación y en sus hipótesis, y muchas veces más allá de ese momento, y que pone en dificultad la primera certidumbre sobre todo en su percepción más ingenua, la de la copia o fotocopia.

Y sin embargo, debe recordarse que el período sobre el que Carlos ha escrito las páginas más admirables es aquel que va del primer peronismo al advenimiento de la revolución argentina $-\mathrm{y}$ yo las encuentro tan admirables al releerlas, creo que son una lectura imprescindible a aconsejar para entender o, mejor, para pensar esos intrincados años argentinos. Pues bien, en esos años Altamirano no es solamente un observador, sino que fue también un testigo. Y él mismo lo ha recordado en ese trabajo tan sentido que se titula "memoria del 69": "solo podría referirme al Cordobazo de memoria, mejor dicho, solo podría referirme a la memoria del Cordobazo", qué bien dicho... Pero, claro está, ello no vale solamente para el caso del Cordobazo.

Carlos no ha decidido elegir, argumentativamente, en un plano más profundo no lo sé, la sugerencia de Dilthey que, como él mismo recuerda bien, fue la de José Luis Romero: la experiencia del mundo como primer criterio para la comprensión del mundo, la erlebnis o experiencia vivida. Decide seguir otro camino, o hablarnos desde otra tradición, o mejor otra retórica: la de la aspiración a una neutralidad axiológica. Así la operación de Carlos para construir esa narrativa que aspira a la neutralidad es doble: tomar distancia de sus recuerdos, primero, tomar distancia de sus interpretaciones partidistas, luego. Que lo haya logrado (en mi perspectiva) obliga a que nos preguntemos si esa ecuanimidad está solo allí.

Esa voluntad irénica, de tomar distancia ¿de dónde emerge? La respuesta por el temperamento nos ayuda hasta ahí, porque todos los que conocemos y hemos escuchado a Carlos sabemos que hay en él, al menos en el Altamirano oral y congresual, una vocación no solo de problematizador, sino de cuestionador y polemista. Y sin embargo, ante la palabra escrita debe haber en Carlos una, muchas pausas, prevenciones, un retorno (fabulo) sobre sus mismos textos buscando el matiz, el equilibrio. A menudo encontramos en los textos de Carlos por momentos un casi abalanzarse sobre algunos autores o figuras para luego incorporar una frase sucesiva que matiza, que quita y da. Nondimanco es una antigua palabra, que Ginzburg ha vuelto popular en el medio académico con su último libro sobre Maquiavelo, Pascal y la casuística. Nondimanco, no obstante, diríamos, sin embargo. Nondimanco. Veamos un ejemplo más que emblemático extraído del libro que se presenta hoy. Dice Carlos: "Victorio Codovilla, un típico y experimentado apparatchik, sin brillo, pero astuto y pragmático". Apparatchik sin brillo, pero...

Hay asimismo una prudencia, el historiador prudente, así como había un rey prudente. ¿Es prudente la palabra? Una vez le apliqué la palabra sensato a mi amigo Boris Fausto, en lo que yo veía un gran elogio, porque la sensatez no abunda en el mundo académico, y creo que no le gustó. Afortunadamente encontré en un texto del mismo Carlos ("Pensar la Argentina entre dos Centenarios") la expresión que buscaba. Cito a Carlos: "A la ciencia del improvisador, Martínez Estrada (referido a Sarmiento), contrapuso la ciencia del baqueano, cuyo depositario era el caudillo, más cauteloso y más próximo a la realidad argentina". Dejemos de lado al caudillo, retengamos al baqueano. Pues, eso es Carlos, un baqueano y los baqueanos saben mejor que los historiadores, sobre todo que los teleológicos, que lo que hay más allá es inquietante, inseguro. Carlos, baqueano. ¿Será por ser correntino? ¿Hay baqueanos en Corrientes?

Creo, sin embargo, que hay algo más o, mejor, algo que se puede formular en términos más doctos. Y ese algo más es un modo de reflexionar sobre la realidad que en Carlos, más allá de su formidable formación teórica, reposa sobre tratar de priorizar la empiria, organizar la empiria. En las líneas finales de un libro de 1908, Filosofía de la Práctica, Croce hizo unas reflexiones que en el fondo ponían en cuestión su primer sistema, pero también hipotecaban los sucesivos, o mejor los dejaban librados a una perpetua inestabilidad. Dice Croce (y perdón, pero yo también tengo mis linajes): 
ningún sistema filosófico puede jamás incluir en sí mismo todo lo filosofable, ningún sistema filosófico es definitivo, porque la vida misma no es jamás definitiva. Un sistema filosófico resuelve un grupo de problemas históricamente dados y prepara las condiciones para poner en posición otros problemas. Así la verdad está siempre circundada de misterio o sea es una ascensión a alturas siempre crecientes que no tienen jamás culminación.

Ahora, yo digo, en esta América nuestra, en esta Argentina nuestra, cómo se puede pensar de otro modo. Si, como alguna vez sostuvo Tulio Halperin entre tantos otros, el presente esclarece el pasado tanto o más que el pasado al presente, cómo no percibir que estamos sobre unas arenas movedizas... Y digo esto consciente de que no solo la Argentina, sino la región está una vez más en un territorio quizá pronosticable pero en el fondo impredecible.

Si la narrativa de Carlos esconde al sujeto cognoscente, elude las adjetivaciones y busca los equilibrios, ¿en dónde está la interpretación? Y claro está, hay una interpretación y una pregunta por el sentido. Carlos no es un anticuario, o una de esas personas que estudian cosas extravagantes bajo la caución de que están preocupados por el destino común. Más aun, él ha dicho claramente que este presente, entendido como presente político, es el que le interesa. Lo dice en este libro al afirmar que no se hizo comunista para leer libros sino para encontrar el modo de transformar el mundo, y por muchas cosas que hayan cambiado, esa voluntad creo que sigue intacta. Por lo demás, quizás (o sin quizás), en algún lugar perduran las viejas lecturas y, desde ellas, el hilo de un historicismo progresivo subsiste en su obra.

Si así estuvieran las cosas, deberíamos volver a leer los textos de Carlos según las sugerencias de Leo Strauss, y mucho antes de Charles Peguy, entre líneas, para encontrar allí, o en el orden de las argumentaciones, o en los comienzos y los finales de los textos, la perspectiva de Carlos. Un autor para lectores inteligentes, me parece (y sin afirmar que yo lo sea). Un autor que, por otra parte, escribe en una prosa elegante y austera pero que contiene acechanzas debajo de un aparente registro uniforme y aunque no proliferen las subordinadas. Una prosa que era expresión, como me dijo una vez de Carlos Tulio Halperin Donghi, que como todos recuerdan era reticente en los elogios, de una cabeza muy bien ordenada. ¿Será el pascaliano espíritu de geometría?

Pero volvamos a abrir Bajo el signo de las masas, en sus primeras páginas, en el apartado que lleva como título "De la revolución nacional a la revolución peronista", está esbozada para mí con claridad la lectura de Altamirano no en torno a un mero debate sobre las ideas, sino en torno a un debate enmarcado en un conflicto entre fuerzas sociales. Más allá de ellos, cuáles son los núcleos problemáticos que organizan su relato: sin excluir otros, quisiera señalar uno que me parece permea muchos de sus textos. Uso el título de uno de ellos: intelectuales y pueblo, pero que aparece declinado de varias maneras, intelectuales y pueblo, élites y masas, pequeña burguesíaperonismo, peronismo verdadero-peronismo empírico (porque el problema de los que Carlos llama peronistas verdaderos no era tan diferente que el que tenía la izquierda clásica con el peronismo empírico o positivo). Una cuestión, por lo demás, que en su mirada antecede largamente como problema al período peronista.

Todavía una última anotación. Unas manos impensadas me hicieron llegar una entrevista a Carlos en la revista "Criterio" del mes de octubre de 2019. Hay ahí, ligeramente en pugna con los entrevistadores, una propuesta de apertura o de diálogo entre distintas tradiciones, porque el diálogo no es ni una frase oportuna, ni una abstracción teórica, sino una práctica, si se prefiere algo más arcaico que apelar a las razones prácticas: un estilo. Un diálogo entre los que se llaman o son llamados populistas, y los que se llaman o son llamados republicanos, y suponiendo que esas etiquetas definan algo más allá de autoadscripciones o atribuciones. No veo qué otra cosa podría hacer un intelectual sino dialogar, aunque sea en la forma de un debate en el que se reconozca al otro como interlocutor. Ello requeriría también, como suele decir Carlos, una nueva versión de "Los conjurados" de Borges: aspirar a ser razonables.

Hace muchos años, no sé cuantos, quizás veinte, me crucé con Carlos, a quien conocía por haber participado juntos en tesis y jurados, en la calle Puán. Me parece recordar que íbamos cada uno en una vereda diferente y que él me hizo un gesto con la mano y yo crucé la calle. No recuerdo mucho más, salvo que me invitó como comentarista a unas jornadas que se realizaban en la Universidad de Quilmes. De allí llegamos hasta aquí, conversando. 